\title{
La capacidad innovadora de la economía mexicana*
}

\author{
The Innovative Capability of the Mexican Economy
}

\author{
Alejandro Mungaray Lagarda, ${ }^{* *}$ Jesús Armando Ríos Flores, ${ }^{* * *}$ José Gabriel \\ Aguilar Barceló ${ }^{* * * *}$ y Martín Arturo Ramírez Urquidy ${ }^{* * * * *}$
}

\section{RESUMEN}

Este estudio analiza el efecto que la capacidad innovadora genera en el nivel de ingreso y en la productividad de algunas variables tecnoeconómicas, como las patentes, las exportaciones en bienes de alta tecnología y en el ingreso por balanza de pagos tecnológica de México. Primero se estima la capacidad innovadora mediante un modelo factorial y, luego, se llevan a cabo las estimaciones con un enfoque de cointegración. Los factores que componen la capacidad para innovar son el entorno productivo, la asimilación tecnológica, la participación universitaria en investigación y desarrollo, y la institucionalidad. Se encuentra que la capacidad innovadora presenta una relación positiva con el nivel de ingreso, negativa con las patentes y el ingreso por balanza de pagos tecnológica e independiente con las exportaciones en bienes tecnológicos al no existir cointegración.

Palabras clave: cointegración, modelo factorial, capacidad innovadora, crecimiento, productividad.

Clasificación JEL: C32, C38, O11, O30.

\section{Abstract}

This study analyzes the effect of innovative capability generated in the level of income and productivity of techno-economic variables such as patents, exports in high-technology goods and income by technological вор Mexico. The first stage was to build innovative capacity through a factor model, while a second held the estimates under a cointegration approach. The factors that make the ability to innovate are productive environment, technological assimilation, university participation in research and development and institutions. It is found that the innovative capability has a positive relationship with the level of income, negative from patents and technology balance of payments and independent with tech goods exports due the absence of cointegration.

Keywords: cointegration, factorial model, innovative capability, growth, productivity. JEL classification: $\mathrm{C} 32, \mathrm{C} 38, \mathrm{O} 11, \mathrm{O} 30$.

\footnotetext{
* Fecha de recepción: 21/09/2013, Fecha de aprobación: 16/07/2015.

** Profesor-investigador de la Facultad de Economía y Relaciones Internacionales de la Universidad Autónoma de Baja California. Correo electrónico: amungaray@baja.gob.mx.

*** Doctorante en Ciencias Económicas de la Facultad de Economía y Relaciones Internacionales de la Universidad Autónoma de Baja California. Correo electrónico: lic.ja.rios@gmail.com. **** Profesor-investigador de la Facultad de Economía y Relaciones Internacionales de la Universidad Autónoma de Baja California. Correo electrónico: gaba@uabc.edu.mx.

***** Profesor-investigador de la Facultad de Economía y Relaciones Internacionales de la Universidad Autónoma de Baja California. Correo electrónico: martinramirez@uabc.edu.mx.
} 


\section{INTRODUCCIÓN}

En la nueva economía global del conocimiento, las capacidades de innovación y aprendizaje se han convertido en una fuente importante de competitividad y crecimiento (Sener y Saridogan, 2011). Sustentadas principalmente en una base científica y tecnológica ligadas al sector productivo, modificando de manera acelerada la composición sectorial de la industria y la dinámica productiva regional. Junto a los cambios en la forma de producir y competir, se ha encontrado que la innovación con impactos económicos y productivos relevantes tiende a estar concentrada en unos pocos países (Furman, Porter y Stern, 2002).

Motivados por esta concentración, surgen una serie de modelos territoriales que realzan las particularidades regionales en la generación de innovación. Por ello, una de las partes centrales del estudio de la innovación se encuentra en las fuentes que determinan la capacidad innovadora (CI), entendida como la capacidad de producción, adquisición y asimilación tecnológica ligada a un espacio determinado, reflejo de las condiciones, inversiones y políticas que sustentan el proceso innovador (Furman, Porter y Stern, 2002; Archibugui y Coco, 2004). Debido a las particularidades de cada entorno regional, conocer las fuentes de la CI proporciona información importante para la generación de políticas tecnológicas pertinentes para el desarrollo productivo de cada región.

Los estudios de la CI se han enfocado al análisis del registro de patentes y el gasto en investigación y desarrollo (GIDE), variables un tanto limitadas cuando se trata de regiones económica e institucionalmente débiles (Albornoz, 2009). Por ejemplo, una patente puede tener distinto grado y rapidez de comercialización de acuerdo con el tipo de tecnología, rama y sector de la economía en el que se produzca, así como de las regulaciones jurídicas imperantes. Además, existen empresas que prefieren mantener en secreto sus descubrimientos tecnológicos y no patentar (Jaffe y Trajtenberg, 2002). El GIDE no es un buen indicador cuando en una determinada industria existe una alta proporción de pequeñas y medianas empresas, puesto que muchas de estas compañías no tienen una cuenta contable propia para las actividades de investigación, aunque las realicen en la propia planta industrial sin contar con un departamento de investigación y desarrollo (ID) específico. En consecuencia, las patentes o el GIDE son imperfectos y, por tanto, se recurre cada vez más a indicadores compuestos que recogen diversos aspectos de la actividad innovadora y enfatizan la importancia de la formación de capital humano, la educación y el entorno económico (Sánchez, García y Mendoza, 2014; Valdez-Lafarga y León-Balderrama, 2015). 
El estudio de la actividad innovadora a partir de estas variables excluye el carácter endógeno de la innovación. Es decir, la que nace por las iniciativas empresariales, de mercado y de competencia, y que se encuentra inmersa en un entorno económico y tecnológico concreto, lo que genera una visión limitada del fenómeno. Por ello, en este estudio se adopta una perspectiva conjunta del entorno económico y tecnológico, ya que en el proceso innovador interactúan una serie de instituciones y organizaciones cuyo grado de integración y desarrollo dependen del ritmo al que se desarrollan e incorporan las innovaciones en las empresas y en el sistema productivo.

La innovación está cobrando un papel central en el desarrollo industrial y competitivo. La dinámica productiva internacional, sustentada en una demanda cambiante y especializada, ha roto con los paradigmas de la producción en masa y transitado hacia sistemas de producción por lotes (Mungaray, 1994). En este contexto, la mayoría de los países apuestan a la innovación mediante el incremento en el GIDE y al fortalecimiento generalizado de las actividades tecnológicas, pero sus resultados no siempre son los esperados (Ríos y Castillo, 2013). Si bien existe consenso de que la CI es crucial para el crecimiento, no existe acuerdo sobre los componentes que la determinan, el peso relativo de cada uno de ellos y las particularidades regionales que limitan sus efectos económicos. A la luz de esta situación, resulta interesante preguntarse: ¿cuáles son los componentes que determinan la CI? Y ¿cuál es el impacto de éstos en el ingreso y en el desempeño innovador?

La mayor parte de los estudios que abordan el tema de la CI se enfocan en los comparativos internacionales, especialmente para los países miembros de la Organización para la Cooperación y el Desarrollo Económicos (OCDE) (Furman, Porter y Stern, 2002; Archibugui y Coco, 2004; Archibugui, Denni y Filipetti, 2009; Porter y Stern, 2010). Para el caso de México, los esfuerzos se han orientado al comparativo estatal y a la formación de indicadores de innovación, dado que las estructuras económicas y tecnológicas son heterogéneas (Ruiz, 2008; Sánchez, García y Mendoza, 2014; Valdez-Lafarga y León-Balderrama, 2015). En la mayoría de los casos se concentran en comparar o caracterizar las regiones y en menor medida en los efectos en la vida productiva y su interacción con el mercado internacional. En este sentido, el objetivo de este trabajo es hacer una estimación estructural y temporal de la CI de México y su impacto en el desempeño económico y tecnológico del país para el período 1990-2010. La hipótesis que se sustenta es que el efecto de la cI en el desempeño técnico y económico es diferenciado y que su impacto es positivo y más que proporcional sobre el ni- 
vel de ingreso, la productividad en la generación de patentes, el ingreso por balanza de pagos tecnológica y la exportación de bienes de alta tecnología.

El trabajo se compone de cinco secciones. En la primera se revisan las principales fuentes de debate en torno a la caja negra del cambio técnico. En la segunda, se presenta la metodología. Ésta se lleva a cabo en dos etapas: para empezar, se utiliza un modelo factorial para la generación del indicador de la CI; después, se utiliza el enfoque de cointegración de Engle y Granger (1987) para establecer las relaciones de la CI con los indicadores tecnoeconómicos. En la sección tres se presentan los datos, mientras que en la cuatro se presenta el análisis de resultados. Finalmente, se establecen las conclusiones.

\section{LA CAJA NEGRA DEL CAMBIO TECNOLÓGICO}

La tecnología siempre ha estado presente en el pensamiento económico, aunque no es sino con Solow (1957) que empieza a cobrar relevancia por su participación en el crecimiento y por su carácter exógeno, al considerarse como un factor "residual" de los factores productivos en la explicación de la tasa de crecimiento (Rosenberg, 1999). Si bien esta visión fue la prevaleciente, no tiene respuestas satisfactorias ante el paradigma productivo actual. Dadas las críticas de la exogeneidad, se inicia la carrera por clarificar el contenido de la "caja negra" de la economía contenida en el "residuo", al que Solow denominó cambio técnico. En este modelo neoclásico con cambio técnico, la tasa de crecimiento de la economía a largo plazo es dirigida por la propia acumulación de progreso tecnológico en el tiempo (Sala-i-Martín, 2000). En consecuencia, la pregunta que surge es: ¿cómo puede acelerarse el progreso tecnológico y con él, el crecimiento?

Algunos teóricos del crecimiento endógeno intentan dar respuesta a lo anterior asignando un papel más activo a los agentes económicos en un escenario en que las empresas generan progreso tecnológico mediante la ID, y donde el crecimiento de la producción de innovaciones depende de la productividad media del capital humano (Griliches, 1979; Romer, 1990; Grossman y Helpman, 1991; Aghion y Howitts, 1992). Sin embargo, para Nelson y Winter (1982), al abordar el tema desde un enfoque sistémico, la innovación posee características exógenas y endógenas de forma conjunta. Por una parte, el efecto endógeno surge del esfuerzo tecnológico de los agentes, mientras que su impacto depende de factores ajenos al agente innovador. Por tanto, los diversos factores que determinan la productividad total no son independientes unos de otros y es necesario considerar los cambios en la naturaleza del acervo de bienes de capital, en la po- 
blación activa y en los efectos de escala como fenómenos que interactúan entre sí (Fagerberg, Srholec y Verspagen, 2010; Autio et al., 2014). Esto cuestiona los modelos lineales de la innovación, tanto los de empuje de la oferta como los de jalón de la demanda, para pasar a modelos interactivos que toman en cuenta, entre otros elementos, la naturaleza de los gastos en ID y de las iniciativas empresariales, resaltando las interrelaciones de los distintos agentes y entornos que intervienen en el proceso (Mungaray y Palacio, 2000).

La innovación va más allá de ID en la medida que comprende todas las fases científicas y técnicas en la solución de los problemas que se plantean a los diversos sectores productivos (RICYT, 2001). Generalmente, la innovación surge como consecuencia de dos factores. El primero por un efecto del incremento del conocimiento a causa de un descubrimiento que permita desarrollar nuevos productos anteriormente desconocidos de una forma más efectiva y barata (Romer, 1990). La segunda forma, acorde a lo propuesto por Blaug (1963), Nelson y Winter (1982) y Lundvall (2010), es mediante la difusión del conocimiento que puede ser aplicado de maneras muy diversas en los diferentes espacios particulares. Este tipo de innovaciones, contrariamente a la percepción tradicional, tiene un impacto igual o más importante que la innovación por la generación del nuevo conocimiento (López-Leyva et al., 2014).

En el plano microeconómico, un descubrimiento científico o una idea creativa es innovación en el momento en que se utiliza para resolver un problema concreto. En consecuencia, el desarrollo tecnológico consiste en su acumulación y actualización permanente en el ámbito de la empresa. En el sentido macroeconómico, el cambio tecnológico está presente cuando las mejoras en una empresa logran convertirse en mejoras para un sector industrial o para la economía en general, lo que se liga directamente con la capacidad global de un país o región para incorporar con rapidez los nuevos paradigmas tecnológicos al sistema económico nacional o regional (Cimoli et al., 2005). Para el análisis de la innovación en el sentido microeconómico, es necesario especificar la forma funcional, las cualidades de los factores, el grado de novedad y su naturaleza, ya que existen diferencias importantes en ellas, ya que las industrias y las empresas son cualitativa y cuantitativamente diferentes (Nelson y Winter, 1982; Acs et al., 2004; Mungaray, Ramírez y Texis, 2005; Audretsch, Keilbach y Lehmann, 2006). En la visión macroeconómica, principalmente de largo plazo, las clasificaciones anteriores resultan irrelevantes (Phelps, 1962; Blaug, 1963; Solow, 1969), ya que en la economía como unidad sistémica, mutuamente dependiente y equilibrada, la innovación individualizada cede su importancia al 
cumulo de innovaciones, su grado de difusión y a la capacidad para asimilar dicho conocimiento.

Según Ayala (2005), la capacidad para incorporar y difundir el progreso técnico depende de las inversiones en instituciones que permitan fortalecer el capital humano y atraer el capital extranjero portador del avance técnico. Por ejemplo, gran parte del avance técnico logrado en Corea del Sur desde la década de los sesenta hasta la de los noventa fue por las inversiones públicas realizadas en la creación y fortalecimiento de capital humano, y la apropiación selectiva de la inversión extranjera directa (Chang, 1993).

Dado que el desembolso en investigación puede llegar a ser alto y los resultados inciertos, no sólo por la concesión de monopolios temporales (patentes, marcas, etc.), sino por la protección de dichas concesiones ante la imitación y la competencia, la protección del capital intelectual se torna fundamental. Un sistema sólido de propiedad intelectual puede tener el efecto de proteger a un innovador, mediante un conjunto de instrumentos legales y técnicos que restringen el uso extendido del nuevo conocimiento, y generando rendimientos extraordinarios por la novedad del producto y la restricción a la entrada de un nicho de mercado. Así, al garantizar la "apropiabilidad" de los inventos, se logra incentivar el proceso creativo para la innovación (Oster, 2000).

Algunos autores evolucionistas trabajan el proceso innovador con el enfoque de sistemas nacionales de innovación a partir de la estructura de producción y del marco institucional de una nación (Fagerberg, Srholec y Verspagen, 2010; Lundvall, 2010; Soete, Verspagen y Baster, 2010). La estructura productiva determina las relaciones que se establecen entre sectores y empresas, así como las rutinas prevalecientes en la producción, la distribución y el consumo.

Mientras el aprendizaje ocurre en una fuerte relación con estas rutinas y relaciones, el marco institucional abarca los centros públicos y privados dedicados a actividades de ID y todas las formas de organización, convenciones y comportamientos prevalecientes en una comunidad, lo que viene a determinar la cualidades del proceso innovador y su productividad (Nelson, 1994). Actualmente, como producto de los procesos de globalización, se han difundido dos visiones relacionadas con el espacio geográfico de la tecnología: una es el "tecnonacionalismo" y la otra es la "tecnoglobalización" (Archibugui y Michie, 1997). Ambas consideran que la única forma de acceder a la tecnología existente en el contexto internacional es a través del nivel de conocimiento del que dispone un país, por lo que el sistema nacional de innovación está presente en esas mediciones. 
Freeman y Pérez (1988) mantienen como premisa que cierto tipo de cambios tecnológicos extienden sus efectos sobre el conjunto de la economía. Estos cambios permiten la aparición de nuevos productos, servicios, sistemas e industrias, que afectan directa o indirectamente a todas las ramas de la economía debido a que influyen en las trayectorias tecnológicas y modifican las estrategias de costos, las condiciones de producción y reconfiguran las necesidades de las sociedades entorno a un paradigma técnico y económico.

Los paradigmas tecnológicos tienen concepciones específicas sobre "cómo se debe hacer las cosas", así como un marco cognoscitivo colectivo. A la vez, también definen modelos básicos de los productos y sistemas de producción que de manera continua se modifican y mejoran. Al igual que en el caso de la ciencia, un paradigma tecnológico implica una definición de los problemas relevantes y de las líneas de investigación, de las necesidades a satisfacer y de los principios científicos y la tecnología a utilizar (Cimoli y Dosi, 1994).

Con esta concepción evolucionista, la dirección del cambio tecnológico se encuentra restringido por el grado de avance de las grandes tecnologías que ya están en uso (Pérez, 1986). En consecuencia, el avance tecnológico de las empresas y organizaciones es una función de los niveles tecnológicos que éstas previamente lograron. En otras palabras, el cambio técnico es en gran medida una actividad acumulativa y justamente por esta característica es que se fundamenta la utilización de la cointegración, ya que refleja la presencia de un equilibrio a largo plazo hacia el cual converge el sistema económico y tecnológico a lo largo del tiempo.

Schumpeter (1939) sostiene que la innovación y el cambio tecnológico son, en lo fundamental, información y conocimiento que se transforma en nuevos productos o procesos, formas de comercialización y organización empresarial, así como novedosas formas de vinculación entre los agentes. Si definimos la tecnología como conocimiento aplicado sistemáticamente a la producción o como resultado de ella, la innovación y el progreso técnico consisten en una adición al conocimiento técnico existente. Dado que la función de producción ya toma en cuenta toda la gama de posibilidades técnicas conocidas, en el sentido de que se practican en alguna parte del sistema, la actividad innovadora debe denotar la adopción de métodos no probados hasta ahora (Schumpeter, 1912; Blaug, 1963). Pero son pocas las innovaciones afortunadas que no tienen una serie de ensayos infortunados y aun la imitación de técnicas ya ensayadas comprende siempre una respuesta creativa. Esta dificultad ha hecho que suela definirse el progreso técnico como cualquier cambio en los métodos de producción, 
independientemente de que los nuevos métodos hayan sido utilizados antes (OCDE y Eurostat, 2005).

Aun con el relajamiento en la definición de innovación es complicado determinar cuándo un descubrimiento científico se transformará en un nuevo producto o proceso, así como sus beneficios comerciales. Ante las dificultades de medición de la innovación, una de las partes centrales de su estudio se encuentra en las fuentes que determinan la CI. Las regiones que dedican grandes recursos a la innovación no necesariamente logran un mayor número de innovaciones efectivas. Asimismo, éstas no están ligadas necesariamente a las regiones con mayores inversiones en innovación. Lo indudable es que las regiones con mejores bases tecnológicas, que incursionan en los segmentos industriales de alta tecnología -en los que se presenta una mayor elasticidad de precios y, por tanto, mayores beneficios-, cuentan con mayores probabilidades de éxito ante los cambios del mercado.

Dentro de las dificultades referentes a la medición de la cI está el determinar cuándo un agente la posee en mayor grado que otro, dado que las estructuras productivas son cuantitativa y cualitativamente diferentes. Por una parte, se podría argumentar que los agentes que obtienen mayores beneficios ligados a los productos innovadores son los que presentan una mayor CI, pero existe evidencia de que una parte importante de las innovaciones en el mercado surge de una serie de eventos no intencionados (Loasby, 2001; Metcalfe, 2002). Por otra parte, el hecho de poseer capacidades tecnológicas desarrolladas, tanto en el monto como en el personal en ID y los centros científicos y tecnológicos, no garantiza que los esfuerzos se traducirán en productos exitosos (Schumpeter, 1928; Nelson y Winter, 1982).

En la literatura teórica y empírica existe una diversidad de estudios que intentan explicar cómo las capacidades tecnológicas y empresariales se transforman en el eje central para la generación de innovaciones y éstas, a su vez, en el motor del desarrollo económico. Stern, Porter y Furman (2000); Archibugui y Coco (2004); Archibugui, Denniy Filipetti (2009), y Porter y Stern (2010) estudian el nivel de la CI en un grupo de países, particularmente los miembros de la OCDE, destacando: 1) la calidad de la infraestructura común de innovación, 2) el entorno innovador específico de los conglomerados, 3) la calidad de los vínculos del sistema en ID, 4) el esfuerzo en ID y el acervo de conocimiento y 5) las habilidades de la fuerza de trabajo (escolaridad). Sus resultados muestran que todas las variables resultan significativas y positivas en la generación de nuevo conocimiento (patentes) para la gran mayoría de países.

Martínez y Baumert (2003) y Buesa et al., (2004), entre otros, reconociendo que existen diferencias regionales y que la innovación es más que sólo 
patentes, presentan -mediante un análisis multivariante- índices de innovación regional que permiten cuantificar en un solo valor la CI de las regiones españolas, los cuales retoman los entornos regional y productivo de la innovación, el papel de las universidades en los sistemas de innovación, el papel de la administración pública y a las empresas innovadoras, siendo estos últimos los factores más importantes del índice.

Stern, Porter y Furman (2000), Martínez y Baumert (2003), Buesa et al., (2004) y Archibugui y Coco (2004) rompen, hasta cierto punto, con la linealidad neoclásica de la innovación y el crecimiento. Por una parte, aceptan el hecho de que el indicador único de patentes está sobreestimado, ya que las capacidades institucionales de los países son significativamente diferentes y, por lógica, los resultados serán diferentes. Este tipo de estudios se acercan más a una idea evolucionista, ya que rescatan la idea del agente económico central, y sus relaciones productivas, y el efecto del sistema regional de innovación como determinantes de los resultados económicos.

En el caso de México, varios estudios han mostrado que los esfuerzos en ID son débiles y que las capacidades de innovación son reducidas (Cimoli, 2000; Aboites y Dutrénit, 2003; Aboites y Soria, 2008). Ruiz (2008); Sánchez, García y Mendoza (2014), y Valdez-Lafarga y León-Balderrama (2015), abordando de forma directa la CI, presentan una caracterización regional, con lo que resaltan el papel de la industria manufacturera, especialmente de alta tecnología, las capacidades científicas, las condiciones de mercado y el efecto institucional.

\section{Metodología}

Dado que la CI se refleja en el movimiento de los indicadores de innovación, es posible argumentar que éste representa el movimiento general de la Cr. Una técnica común para capturar este tipo de cambios es el modelo factorial (MF) (Martínez y Baumert, 2003; Buesa et al., 2004; Sánchez, García y Mendoza, 2014; Valdez-Lafarga y León-Balderrama, 2015). Su función es encontrar el número mínimo de variables que expliquen el máximo de información contenida en una muestra, simplificando las relaciones entre un conjunto de variables observables, donde puede ser cualquier número finito. En particular, se trata de encontrar factores comunes que expliquen de modo suficiente las variables originales observables, además de evitar los juicios a priori sobre la participación que debe tener cada indicador que compone la CI. Diversos autores destacan el hecho de que la innovación incluye elementos no susceptibles de medición (Albornoz, 2009), o 
20 ECONOMÍA: TEORÍA Y PRÁCTICA • Nueva Época, número 43, julio-diciembre 2015

Cuadro 1. Variables sujetas a la aplicación del MF

\begin{tabular}{|c|c|c|}
\hline Indicador & Medida & Fuente \\
\hline SNICa & $\begin{array}{l}\text { Miembros del sistema nacional de investigadores en cien- } \\
\text { cias naturales y exactas, de la salud, agropecuarias, e inge- } \\
\text { niería y tecnología por cada millón de habitantes }\end{array}$ & CONACYT \\
\hline SNICb & $\begin{array}{l}\text { Miembros del sistema nacional de investigadores en cien- } \\
\text { cias sociales y humanidades y en educación y humanidades } \\
\text { por cada millón de habitantes }\end{array}$ & CONACYT \\
\hline GIDEP & GIDE público/GIDE total & CONACYT \\
\hline GIDEPRI & GIDE privado/GIDE total & CONACYT \\
\hline GIDEU & GIDE universitario/GIDE total & CONACYT \\
\hline IBAT & $\begin{array}{l}\text { Importaciones de bienes de alta tecnología como } \\
\text { porcentaje del PIB }\end{array}$ & Banco Mundial \\
\hline EBPT & $\begin{array}{l}\text { Egresos por regalías, asistencia técnica, etc., como porcen- } \\
\text { taje del PIB }\end{array}$ & Banco Mundial \\
\hline IMPI & $\begin{array}{l}\text { Patentes concedidas en el Instituto Mexicano de la Propie- } \\
\text { dad Industrial (IMPI) por cada millón de habitantes }\end{array}$ & OMPI \\
\hline EBAT & $\begin{array}{l}\text { Exportaciones de bienes de alta tecnología como porcen- } \\
\text { taje del PIB }\end{array}$ & Banco Mundial \\
\hline IBPT & $\begin{array}{l}\text { Ingresos por regalías, asistencia técnica, etc. como porcen- } \\
\text { taje del PIB }\end{array}$ & Banco Mundial \\
\hline UI & Usuarios de internet por cada 100 habitantes & Banco Mundial \\
\hline CENTROS & $\begin{array}{l}\text { Centros CONACYT, Consejos Estatales de Ciencia y Tecnolo- } \\
\text { gía y fundaciones Produce por estado por cada millón de } \\
\text { habitantes }\end{array}$ & $\begin{array}{l}\text { CONACYT y Fundación } \\
\text { Produce }\end{array}$ \\
\hline IPS & Porcentaje del software que es adquirido por piratería & $\begin{array}{l}\text { Business Software } \\
\text { Aliance }\end{array}$ \\
\hline $\mathrm{TI}$ & Tasa de interés activa & Banco Mundial \\
\hline PIB & PIB en billones de dólares & Penn World Table \\
\hline РОВ & Población en millones & Penn World Table \\
\hline ISO & $\begin{array}{l}\text { Establecimientos con certificados ISO por cada millón de } \\
\text { habitantes }\end{array}$ & CONACYT \\
\hline IPM & Índice de productividad manufacturera base 1993 & INEGI \\
\hline IED & Inversión extranjera directa como porcentaje del PIB & Banco Mundial \\
\hline
\end{tabular}

Fuente: Elaboración propia. 
que indicadores como las patentes han sido concebidas para su uso en un marco legal y no con el propósito específico de medir la innovación. Con el objetivo de capturar de manera sistémica la CI, en este trabajo se consideran una diversidad de variables (cuadro 1) de naturaleza económica y tecnológica, tanto de esfuerzo como de resultado y de interacción internacional, con datos anuales para México en el periodo 1990-2010. A diferencia de los trabajos de Sánchez, García y Mendoza (2014), y Valdez-Lafarga y León-Balderrama (2015), aquí se consideran no sólo los indicadores tecnológicos, sino ciertos resultados económicos de dichas tecnologías y además se diferencia por sectores de ejecución del GIDE.

Sea $X$ la matriz de variables originales, donde la varianza es una medida de la información que contiene cada una, la combinación lineal de las variables originales puede expresarse como sigue:

$$
F_{1 i}=u_{11} X_{1 i}+u_{12} X_{2 i}+\ldots+u_{1 p} X_{p i}
$$

De manera abreviada se puede expresar (1) como $F_{l}=X u_{l}$, donde $u_{l}$ es el vector que permite obtener la combinación lineal. Dado que el primer factor es la combinación lineal de las variables originales de varianza máxima, $u_{l}$ debe tener valor unitario para que la varianza del primer factor $F$ sea máxima.

Como la varianza del factor $F$ es:

$$
V\left(F_{i}\right)=\frac{\sum_{i=1}^{n} C_{1 i}^{2}}{n}=\frac{1}{n} F_{i}^{\prime} F_{i}=\frac{1}{n} u_{i}^{\prime} X^{\prime} X u_{i}=u_{i}^{\prime}\left[\frac{1}{n} X^{\prime} X\right] u_{i}=u_{i}^{\prime} V u_{i}^{\prime}
$$

Se puede resumir el problema del factor de la manera siguiente:

$$
\operatorname{Max} V\left(F_{i}\right)=u_{i}^{\prime} V u_{i}^{\prime} \operatorname{Max}
$$

$$
\text { Sujeta a: } \quad \sum_{j=1}^{p} u_{1 i}^{2}=u_{1}^{\prime} u_{1}=1
$$

Resolviendo el lagrangiano de la ecuación (3) se obtiene:

$$
(V-\lambda I) u_{1}=0
$$

Donde $I$ es la matriz identidad y $u_{1}$ es el vector propio de la matriz de varianza y covarianza de los datos originales. Como se busca la varianza máxima, se elige el vector propio con mayor valor asociado. El primer factor $F_{1}$ se obtiene haciendo $F_{l}=V u_{l}$, donde $u_{l}$ es el vector propio de la matriz varianza-covarianza con 
mayor valor asociado. El segundo factor de acuerdo con (2) estará dado por $V\left(C_{h}\right)=u^{\prime}{ }_{h} V u_{h}=\lambda_{h}$ (Pérez, 2006). Para el cálculo de la CI la varianza total se reescala a la unidad, por lo que los pesos relativos de cada factor se modifican en el mismo sentido.

La aplicación del MF requiere desarrollar cuatro fases interdependientes. En primer lugar se realiza el cálculo de una matriz que indique la varianza de las variables estudiadas; luego se lleva a cabo la extracción de los factores que agrupan un conjunto de variables con características comunes mediante el método de componentes principales, en que los factores obtenidos son los autovalores de la matriz de correlaciones reescalados; en tercer lugar se rota la matriz de componentes con el método VARIMAX, que permite una redistribución de la varianza de los primeros factores a los últimos, obteniendo un patrón de factores simple y significativo en que tanto la varianza total explicada por los factores, como las comunalidades de cada una de las variables, quedan inalteradas; y finalmente se estiman las puntuaciones de las variables en los factores.

Para corroborar la pertinencia del MF, se llevaron a cabo las pruebas de KMO (Kaiser, Meyer y Olkin) y de Bartlett. La adecuación muestral permite su aplicación, ya que el KMO es de 0.719 y la prueba de esfericidad de Bartlett tiene una significación menor que 0.05 . Los resultados de la matriz de componentes rotados proporcionan información sobre la ubicación de cada una de las variables del cuadro 1 para establecer las agrupaciones (cuadro 2) y las bases para determinar el indicador de la ci y su taxonomía.

Cuadro 2. Composición de la Cl: taxonomía y pesos

\begin{tabular}{ccccc}
\hline \multirow{2}{*}{ Características } & $\begin{array}{c}\text { 1. Entorno } \\
\text { productivo (EP) }\end{array}$ & $\begin{array}{c}\text { 2. Asimilación } \\
\text { tecnológica (AT) }\end{array}$ & $\begin{array}{c}\text { 3. Participación de } \\
\text { las universidades } \\
\text { en ID (PUID) }\end{array}$ & $\begin{array}{c}4 . \\
\text { Institucionalidad } \\
\text { (INS) }\end{array}$ \\
\cline { 2 - 5 } Variables & $\begin{array}{c}\text { IBPT, SNICb, } \\
\text { SNICa, GIDEPRI, } \\
\text { UI, TI, PIB, POB, } \\
\text { ISO, IPM. }\end{array}$ & $\begin{array}{c}\text { IMPI, EBAT, EBPT, } \\
\text { GIDEP. IBAT, } \\
\text { CENTROS, IED. }\end{array}$ & GIDEU. & IPS. \\
\hline $\begin{array}{c}\text { Varianza total } \\
\text { explicada }\end{array}$ & $56.63 \%$ & $20.13 \%$ & $5.42 \%$ \\
$\begin{array}{c}\text { Varianza total } \\
\text { estandarizada }\end{array}$ & $64.02 \%$ & $22.75 \%$ & $7.08 \%$ & $6.12 \%$ \\
\hline
\end{tabular}

Fuente: Elaboración propia. 
Con el MF se logra explicar el $88.45 \%$ de la varianza total de las variables. El factor más significativo es el de entorno productivo que representa el $55.63 \%$ del modelo, mientras que el factor asociado a la asimilación tecnológica representa el $20.13 \%$. Los resultados son consistentes con la literatura al asociar como factor principal para el crecimiento y desarrollo tecnológico al entorno productivo como generador y demandante de innovaciones. En segundo término, el grado de asimilación tecnológica proporciona las capacidades de aprendizaje y transformación del nuevo conocimiento. Los factores de participación universitaria e institucional resultan con una participación escasa al momento de determinar la CI.

La construcción de la CI se realiza con los $k$ factores ponderados por su varianza de la forma:

$$
C I_{t}=\sum_{k=1}^{k} F_{k t} \beta_{k}
$$

donde $C I$ indica la capacidad innovadora en el momento $t, F$ es el factor $k$ que representa los valores reescalados de las variables originales y $\beta$ es la carga de cada factor dada su varianza total estandarizada de forma que $\sum_{k=1}^{k} \beta_{k}=1$.

El indicador de la CI y sus factores son los ejes centrales para la explicación tanto para el ingreso como para la productividad en la generación de innovaciones. Una de las cualidades del MF es que los factores y sus variables son incorrelacionados, lo que garantiza la exogeneidad (Pérez, 2006), cuestión importante para la ejecución del modelo econométrico. Con la combinación de ambos métodos se busca la interacción entre economía y tecnología para representar la trayectoria tecnológica del sistema innovador mexicano.

Dado que la mayor parte de las series temporales no son estacionarias, las técnicas de regresión basadas en datos no estacionarios tienden a producir resultados espurios. Sin embargo, las series no estacionarias pueden estar cointegradas si alguna combinación lineal de las series llega a ser estacionaria. Por lo tanto, las series cointegradas no se separarán muy lejos unas de otras debido a que están enlazadas en el largo plazo. Dentro de las pruebas para determinar la estacionariedad de una variable se encuentran las pruebas de raíces unitarias como las de Dickey-Fuller, Phillips-Perron y la KPss, con las cuales se determina el orden de integración de las variables.

Una vez determinado el orden de integración de las variables se realiza un ejercicio de cointegración para la contrastación de la hipótesis bajo la metodología de Engle y Granger (1987), de la siguiente forma: 


$$
\operatorname{LogIT} T_{j t}=\alpha_{1 j}+\alpha_{2 j} \log C I_{t}+\varepsilon_{j}
$$

donde $I T$ representa los indicadores tecnoeconómicos $j$, que son el nivel de ingreso, la productividad en la generación de patentes, ingreso por balanza de pagos tecnológica y en las exportaciones en bienes de alta tecnología, en el momento $t$. CI es la capacidad innovadora en el momento $t$, el cual es calculado mediante el MF. El coeficiente $\alpha_{1 j}$ representa una constante, $\alpha_{2 j}$ representa la elasticidad asociada a la variable CI y $\varepsilon_{j}$ representa el error estocástico.

Uno de los principales criterios de generación de valor agregado en la producción de bienes y servicios de las empresas, se determina por los esfuerzos que hacen para desarrollar tecnología propia, producir nuevos bienes y servicios y mejorar sustancialmente los existentes. Las empresas que destinan recursos en estos rubros, frecuentemente los ven reflejados en un mayor valor agregado de su producción, un mejor posicionamiento en los mercados doméstico e internacional y en una derrama positiva de nuevos conocimientos y desarrollos. Esto beneficia colateralmente a otros sectores productivos debido a la derrama, o bien, presiona a otras empresas a incrementar sus niveles de competitividad para permanecer en el mercado. Entonces, a medida que la CI aumenta, la productividad de los indicadores de la innovación aumentará en el mismo sentido, por lo que se espera que los estimadores sean positivos y significativos.

\section{ANÁLISIS DE DATOS}

En el cuadro 3 se presentan las variables tecnoeconómicas para la ecuación (5). Dentro de los indicadores de innovación relacionados con el criterio anterior están las exportaciones de bienes de alta tecnología (EBAT), los ingresos por balanza de pagos tecnológica (IBPT) y la concesión de patentes a residentes mexicanos en el Instituto Mexicano de la Propiedad Industrial (IMPI) y en la United States Patent and Trademark Office (USPTO), todos divididos por el GIDE, que en cierta forma reflejan su productividad, junto con el PIB y la CI que se obtuvo con el MF. El PIB se toma de Heston, Summers y Aten (2010), presentado en dólares per cápita de 2005. Las patentes utilizadas son las concedidas en el IMPI y en la USPTO. Mientras El IBPT y las EBAT se toman del Consejo Nacional de Ciencia y Tecnología (CONACYT). Para estandarizar las variables monetarias se estiman en términos de proporción del PIB y luego se multiplican por el PIB per cápita con base en Heston, Summers y Aten (2010). 
Cuadro 3. Indicadores tecnoeconómicos de México

\begin{tabular}{|c|c|c|c|c|c|c|}
\hline Periodo & $\begin{array}{c}\text { PIB } \\
\text { per cápita }\end{array}$ & $\begin{array}{l}\text { Productividad } \\
\text { de patentes } \\
\text { concedidas en } \\
\text { el IMPI } \\
\text { (IMPI/GIDE) }\end{array}$ & $\begin{array}{c}\text { Productividad } \\
\text { de patentes } \\
\text { concedidas } \\
\text { en la USPTO } \\
\text { (USPTO/GIDE) }\end{array}$ & $\begin{array}{c}\text { Productividad } \\
\text { de las EBAT } \\
\text { (EBAT /GIDE) }\end{array}$ & $\begin{array}{c}\text { Productividad } \\
\text { del IBPT } \\
\text { (IBPT /GIDE) }\end{array}$ & $\mathrm{Cl}$ \\
\hline 1990 & 6392.87 & 0.103 & 0.025 & 2.728 & 0.057 & 17.588 \\
\hline 1991 & 6730.63 & 0.089 & 0.020 & 1.595 & 0.054 & 17.718 \\
\hline 1992 & 7019.63 & 0.164 & 0.024 & 2.383 & 0.052 & 18.220 \\
\hline 1993 & 7189.18 & 0.242 & 0.032 & 3.003 & 0.067 & 18.106 \\
\hline 1994 & 7521.75 & 0.145 & 0.022 & 2.776 & 0.053 & 18.573 \\
\hline 1995 & 7013.96 & 0.073 & 0.020 & 3.918 & 0.057 & 19.019 \\
\hline 1996 & 7358.41 & 0.054 & 0.018 & 6.308 & 0.057 & 20.048 \\
\hline 1997 & 7836.00 & 0.044 & 0.018 & 6.924 & 0.051 & 20.929 \\
\hline 1998 & 8072.17 & 0.047 & 0.019 & 7.169 & 0.046 & 21.683 \\
\hline 1999 & 8469.40 & 0.033 & 0.021 & 7.346 & 0.012 & 22.352 \\
\hline 2000 & 9230.75 & 0.033 & 0.022 & 10.001 & 0.013 & 23.479 \\
\hline 2001 & 9348.25 & 0.032 & 0.022 & 9.202 & 0.011 & 24.373 \\
\hline 2002 & 9499.11 & 0.034 & 0.023 & 7.845 & 0.016 & 26.645 \\
\hline 2003 & 9689.20 & 0.027 & 0.019 & 7.001 & 0.017 & 26.397 \\
\hline 2004 & 10224.82 & 0.034 & 0.018 & 7.809 & 0.010 & 27.792 \\
\hline 2005 & 11964.79 & 0.022 & 0.014 & 6.190 & 0.012 & 28.767 \\
\hline 2006 & 12922.23 & 0.022 & 0.011 & 6.612 & 0.013 & 26.685 \\
\hline 2007 & 13626.70 & 0.032 & 0.009 & 6.806 & 0.015 & 31.615 \\
\hline 2008 & 14167.88 & 0.026 & 0.007 & 6.096 & 0.011 & 32.868 \\
\hline 2009 & 12887.42 & 0.029 & 0.008 & 5.778 & 0.013 & 32.129 \\
\hline 2010 & 13900.00 & 0.028 & 0.012 & 5.407 & 0.011 & 33.701 \\
\hline Media & 9574.53 & 0.06 & 0.02 & 5.85 & 0.03 & 24.22 \\
\hline Máximo & 14167.88 & 0.24 & 0.03 & 10.00 & 0.07 & 33.70 \\
\hline Mínimo & 6392.87 & 0.02 & 0.01 & 1.60 & 0.01 & 17.59 \\
\hline Des. Est. & 2620.55 & 0.06 & 0.01 & 2.30 & 0.02 & 5.43 \\
\hline
\end{tabular}

Fuente: Elaboración propia con datos de CONACYT, IMPI, USPTO, Banco Mundial y Heston, Summers y Aten (2010).

El PIB presenta una tendencia positiva, con altibajos en las crisis de 1994-1995 y la de 2008-2009, mientras que el año de mayor ingreso es 2008 con USD 14 167.88. Los periodos más productivos en la generación de patentes tanto en el IMPI como en la USPTO, son de 1990 a 1994. En promedio se genera 0.06 y 
0.02 patentes por cada millón de dólares gastados en ID en cada oficina respectivamente. El IBPT presenta una productividad baja, pues en promedio por cada dólar invertido se obtienen USD 0.03 en el comercio de conocimiento, lo cual es limitado respecto a la inversión que se realiza. El indicador que ha tenido una

\section{Gráfica 1. Evolución y relaciones entre los indicadores tecnoeconómicos de México}
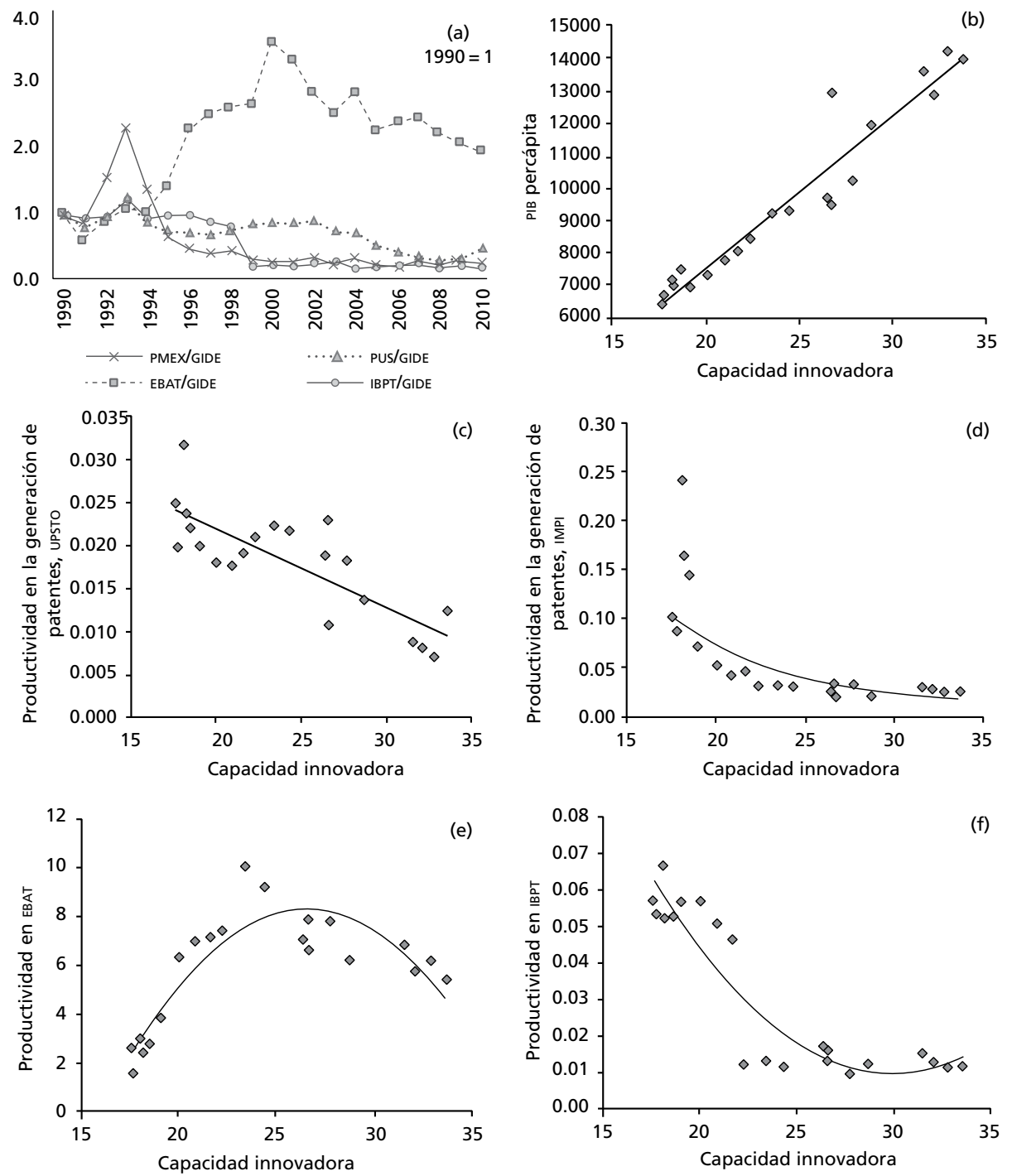

Fuente: Elaboración propia con datos del cuadro 3. 
dinámica diferente es el de las EBAT. Por cada dólar que se invierte se generan en promedio USD 5.85, incluso en el año 2000 que es el máximo registrado, se obtuvieron USD 10 por dólar invertido. En la Cr la dinámica ha sido creciente, con una caída significativa en 2006. En el cuadro 4 se presentan las pruebas de raíces unitarias para los indicadores técnicos y económicos, así como para los factores que componen la CI.

En la gráfica 1 se presenta la evolución de las variables presentadas en el cuadro 3 tomando como base 1990. Así mismo, se presentan de forma ilustrativa las posibles correlaciones entre CI e indicadores tecnoeconómicos.

En el panel (a) de la gráfica 1 se aprecia cómo los indicadores presentan una tendencia negativa. Tanto la productividad en la generación de patentes como la productividad en la balanza de pagos tecnológica son decrecientes, ya que el GIDE ha aumentado y los resultados no han mejorado. En el caso de la productividad en las EBAT, aunque ha comenzado a decrecer significativamente, sigue siendo muy superior al resto. Para el caso del panel (b), donde se relaciona la CI y el PIB per cápita, la correlación es positiva, lo que sin duda presenta evidencia contradictoria, comparada con los paneles (c) (d) (e) y (f) sobre las capacidades tecnológicas y económicas.

Cuadro 4. Pruebas de raíces unitarias para las variables seleccionadas

\begin{tabular}{|c|c|c|c|c|c|c|c|}
\hline \multirow{2}{*}{ Variable } & \multicolumn{2}{|c|}{ Dickey-Fuller } & \multicolumn{2}{|c|}{ Phillips-Perron } & \multicolumn{2}{|c|}{ KPSS } & \multirow{2}{*}{$\begin{array}{l}\text { Orden de } \\
\text { integración }\end{array}$} \\
\hline & Nivel & 1ra. Diff. & Nivel & 1ra. Diff. & Nivel & 1ra. Diff. & \\
\hline PIB per cápita & -0.23 & $-6.21 *$ & -1.86 & $-4.00 *$ & $0.14 *$ & $0.06^{*}$ & $\mathrm{I}(1)$ \\
\hline EBAT/GIDE & -0.84 & $-4.78^{*}$ & -0.74 & $-7.05^{*}$ & $0.15^{*}$ & 0.10 * & $\mathrm{I}(1)$ \\
\hline IBPT/GIDE & -1.83 & $-4.72^{*}$ & -1.85 & $-4.72 *$ & $0.11 *$ & $0.07 *$ & $\mathrm{I}(1)$ \\
\hline IMPI/GIDE & $-3.33^{*}$ & $-8.91 *$ & -2.24 & $-3.22 *$ & $0.12^{*}$ & $0.13^{*}$ & $\mathrm{I}(1)$ \\
\hline USPTO/GIDE & -2.14 & $-4.33^{*}$ & -2.46 & $-4.33^{*}$ & 0.10 * & $0.14 *$ & $\mathrm{I}(1)$ \\
\hline $\mathrm{Cl}$ & $-4.14^{\star}$ & $-5.18^{*}$ & $-4.67^{*}$ & $-13.75^{\star}$ & 0.11 & $026^{*}$ & $\mathrm{I}(1)$ \\
\hline$E P$ & -2.74 & $-5.68^{*}$ & -1.61 & $-15.18^{*}$ & 0.16 & 0.25 & $\mathrm{I}(1)$ \\
\hline$A T$ & -2.62 & $-5.29 *$ & 0.89 & $-11.29 *$ & 0.17 & 0.35 & $\mathrm{I}(1)$ \\
\hline PUID & -1.84 & $-6.28^{*}$ & -0.5 & $-10.41 *$ & 0.17 & 0.27 & $\mathrm{I}(1)$ \\
\hline INS & -3.06 & $-3.90 *$ & -2.99 & $-12.95^{*}$ & $0.18^{*}$ & 0.24 & $\mathrm{I}(1)$ \\
\hline
\end{tabular}

Fuente: Elaboración propia.

Nota: Los estadísticos con * representan la significación al $5 \%$ que rechaza la prueba de no estacionariedad. Las variables en negrillas están expresadas en logaritmos. 
Todas las variables del modelo son estacionarias en primera diferencia, lo que garantiza la correcta identificación de la existencia de relaciones estables de largo plazo entre las variables. Ello permite identificar los componentes tendenciales de las series y determinar si existe un comportamiento común entre ellos.

\section{RESULTADOS}

En el cuadro 5 se presentan los resultados de las estimaciones de los indicadores de productividad tecnoeconómicos y PIB per cápita con respecto a la CI, previamente transformadas en logaritmos. Se observan las elasticidades entre los indicadores mencionados, el grado de explicación del modelo medido por el $R^{2}$ estimado y la especificación de la presencia de cointegración entre las variables. Todos los indicadores resultaron significativos al 5\%, no así sus signos y la estabilidad de su relación.

Cuadro 5. La Cl y los indicadores tecnoeconómicos de México

\begin{tabular}{cccccc}
\hline & PIB per cápita & $\begin{array}{c}\text { Productividad } \\
\text { de las EBAT }\end{array}$ & $\begin{array}{c}\text { Productividad } \\
\text { del IBPT }\end{array}$ & $\begin{array}{c}\text { Productividad } \\
\text { de patentes } \\
\text { concedidas en } \\
\text { el IMPI }\end{array}$ & $\begin{array}{c}\text { Productividad } \\
\text { de patentes } \\
\text { concedidas en } \\
\text { USPTO }\end{array}$ \\
\hline$\alpha$ & 5.48 & -2.75 & 5.58 & 5.22 & 0.46 \\
Cl & 1.15 & 1.39 & -2.94 & -2.61 & -1.43 \\
$R^{2}$ & $(17.07)$ & $(3.51)$ & $(-7.68)$ & $(-6.46)$ & $(-5.72)$ \\
\hline Cointegración & 0.93 & 0.39 & 0.75 & 0.68 & 0.63 \\
\hline
\end{tabular}

Fuente: Elaboración propia. Entre paréntesis se presentan las pruebas estadísticas

Existe cointegración en el sentido de que existe un movimiento común de la CI con el PIB per cápita y la productividad en IBPT, IMPI y USPTO, no así para las EBAT. Resulta interesante la elasticidad negativa presente en los indicadores de conocimiento tecnológico y la no cointegración con la variable ligada a la presencia de tecnología integrada a los bienes exportados. Al comparar las estimaciones del cuadro 5 con los paneles (c) (d) y (f) de la gráfica 1, se aprecia claramente la razón de los signos negativos. Típicamente se espera que a medida que aumenta la capacidad innovadora de un país, la productividad en la genera- 
ción de bienes intensivos en conocimiento lo haga en el mismo sentido. En consecuencia, los resultados generados se tornan contradictorios.

En el cuadro 6 se realizan las estimaciones directamente con los cuatro componentes de la ci para ver el impacto de cada uno, verificar las causas de no cointegración y las elasticidades negativas con los indicadores de productividad. Al realizar las estimaciones bajo un modelo semilogaritmico, ya que existen valores no positivos en los factores, todos los indicadores resultan significativos al $5 \%$ y presentan cointegración entre el PIB y los factores.

Cuadro 6. Componentes de la cl e indicadores tecnoeconómicos de México

\begin{tabular}{|c|c|c|c|c|c|}
\hline & PIB per cápita & $\begin{array}{l}\text { Productividad } \\
\text { de las EBAT }\end{array}$ & $\begin{array}{l}\text { Productividad } \\
\text { del IBPT }\end{array}$ & $\begin{array}{c}\text { Productividad } \\
\text { de patentes } \\
\text { concedidas en } \\
\text { el IMPI }\end{array}$ & $\begin{array}{c}\text { Productividad } \\
\text { de patentes } \\
\text { concedidas en } \\
\text { USPTO }\end{array}$ \\
\hline$\alpha$ & 7.71 & -0.07 & -0.47 & 0.11 & -2.68 \\
\hline EP & $\begin{array}{c}0.06 \\
(6.32)\end{array}$ & $\begin{array}{c}0.15 \\
(5.17)\end{array}$ & $\begin{array}{l}-0.08 \\
(-7.55)\end{array}$ & & \\
\hline AT & $\begin{array}{l}-0.02 \\
(-2.32)\end{array}$ & $\begin{array}{c}0.31 \\
(6.02)\end{array}$ & $\begin{array}{l}-0.09 \\
(-3.07)\end{array}$ & & $\begin{array}{c}0.07 \\
(5.04)\end{array}$ \\
\hline PUID & $\begin{array}{c}0.08 \\
(3.38)\end{array}$ & & & & \\
\hline INS & & $\begin{array}{l}-0.36 \\
(-3.47)\end{array}$ & & $\begin{array}{l}-0.27 \\
(-7.46)\end{array}$ & $\begin{array}{c}-0.12 \\
(-5.77)\end{array}$ \\
\hline$R^{2}$ & 0.96 & 0.89 & 0.76 & 0.74 & 0.75 \\
\hline Cointegración & sí & sí & sí & sí & sí \\
\hline
\end{tabular}

Fuente: Elaboración propia. Entre paréntesis aparecen las pruebas estadísticas.

Con la nueva especificación, donde la ci se sustituye por los componentes que la constituyen, se encuentran las razones de los signos que se reflejan en la regresión y el impacto diferente de cada componente. Los factores EP, AT y PUID se encuentran cointegrados con el PIB, resaltando de forma interesante que la participación universitaria en la generación de nuevo conocimiento, presenta un mayor efecto en el nivel de ingreso que el propio entorno productivo. El efecto de la AT es negativo aunque cercano a cero, reflejando un esfuerzo desaprovechado en forma global por el sistema económico. 
En el caso de la productividad de las patentes en el IMPI y la USPTO, el efecto institucional es contundente. El incentivo económico del inventor mexicano para el registro de la propiedad intelectual es nulo, incluso negativo por el costo de patentar y posiblemente por el temor de las imitaciones no controladas del descubrimiento. El hecho de que la elasticidad menos negativa sea en la USPTO, es reflejo de la fortaleza estadounidense en la protección de la propiedad intelectual.

Ligada de manera estrecha con el nulo incentivo por patentar, la IBPT se tornó poco dinámica considerando que dentro de sus principales componentes se encuentra el ingreso por la venta y derechos de uso de una patente. El efecto negativo del EP y la AT puede ser reflejo de la falta de capacidad del sector empresarial para la generación de bienes intangibles y con alto contenido tecnológico. Dentro de las tendencias globales se encuentra el hecho que los países desarrollados comienzan a reorientar su sector productivo hacia los bienes intangibles pero tecnológicamente sofisticados, pasando los procesos manufactureros a los países en vías de desarrollo. Prueba de ello es que dentro de los indicadores tecnoeconómicos con productividad positiva se encuentran las EBAT de origen manufacturero.

La productividad en las EBAT se ha sustentado en el EP y la AT. Los bienes tecnológicos exportables son generados principalmente por las empresas manufactureras ligadas a una cadena de valor de carácter internacional, por lo que las capacidades productivas y la asimilación del conocimiento externo se tornan fundamentales. En los últimos años, con la reconfiguración productiva a nivel internacional, la tendencia de la productividad comienza a revertirse debido a que una gran cantidad de empresas migran hacia regiones donde el factor trabajo es menos costoso, en busca de optimizar su función de producción. En consecuencia el incremento en el GIDE no se ha reflejado en un mayor rendimiento de las EBAT.

Cada una de las estimaciones genera evidencia de un efecto sistémico de la economía. El entorno productivo no genera incentivos económicos para la transformación productiva hacia la generación de bienes tecnológicos intangibles ligados a la propiedad intelectual. Al no garantizar la propiedad intelectual, el entorno institucional desincentiva el proceso creativo para generar innovaciones.

\section{CONCLUSIONES}

Se parte del hecho de que la innovación de productos y procesos es tan importante como el entorno donde ésta se genera y difunde. En el plano microeconómico 
un descubrimiento científico o una idea creativa son innovaciones desde el momento mismo en que son utilizados para resolver un problema concreto. En consecuencia, el desarrollo tecnológico consiste en su actualización permanente en el ámbito de la empresa. En el plano macroeconómico, el cambio tecnológico se hace presente cuando las mejoras en una empresa logran convertirse en mejoras para todo un sector industrial o para la economía en general. De esta manera, se vincula directamente con la capacidad global de un país o región para incorporar con rapidez los nuevos paradigmas tecnológicos al sistema económico nacional o regional.

Los factores que determinan la capacidad innovadora en México son: 1) el entorno productivo, que refleja el entorno empresarial y competitivo; 2) la asimilación tecnológica, como la eficiencia en el aprovechamiento de la tecnología adquirida del exterior; 3) la institucionalidad, vista como la capacidad del sector público para ejercer la protección de los derechos de propiedad, y 4) la participación universitaria en ID. Los resultados confirman la hipótesis de que la CI tiene un impacto positivo y más que proporcional en el nivel de ingreso y en la productividad de las exportaciones de bienes de alta tecnología, rechazándose para la productividad de concesiones de patentes a residentes mexicanos en la oficina de patentes de México y Estados Unidos, así como para los ingresos de la balanza de pagos tecnológica, pues si bien las series se cointegran, presentan elasticidades negativas.

En la mayoría de los estudios que analizan la CI (Sánchez, García y Mendoza, 2014; Valdez-Lafarga y León-Balderrama, 2015), se encuentran efectos positivos. A diferencia de este tipo de estudios, aquí se presentan los indicadores tecnológicos ponderados por el GIDE, reflejando hasta cierto punto la productividad de cada indicador. Si bien muestra efectos positivos dados por su crecimiento, no responde en la misma proporción en la que se incrementa el GIDE.

De acuerdo con los factores que integran la capacidad innovadora y el impacto de cada uno, es posible plantear algunas nociones de política económica y tecnológica para México. En materia tecnológica, si bien la participación de las universidades en los procesos innovadores ha sido creciente, requiere ser mayor pues el conocimiento tecnológico resulta fundamental para la creación de ventajas competitivas en estructuras productivas cada vez más cambiantes. En materia de institucionalidad, un sólido sistema de propiedad intelectual permite incentivar a los científicos y tecnólogos para seguir investigando. En todos los casos, es necesario fortalecer la participación pública en apoyo a la generación y asimilación de tecnologías. 
A medida que se fortalezca el sistema de patentes, se incentivará el desarrollo tecnológico interno con aplicaciones masivas para la economía y para su comercialización internacional. El hecho de que algunos de los componentes resulten irrelevantes de forma individual demuestra la presencia del efecto sistémico de la economía. Por ello, para lograr el impacto esperado de la capacidad innovadora en la economía, es necesario gestionar cada uno de los componentes de forma conjunta.

El número de patentes expedidas para una empresa rinde incrementos sustanciales en su valor de mercado, ya sea por la generación y utilización de nuevos productos o procesos, o por el efecto estratégico de limitar las oportunidades potenciales a sus rivales comerciales para la utilización de una nueva tecnología. Si bien el efecto estratégico de dicho procedimiento escapa al objetivo de este trabajo, la baja productividad en generación de patentes, acompañada de una alta productividad en las exportaciones de bienes de alta tecnología, proporciona indicios de que las empresas se orientan más al secreto industrial que a patentar y presentan una fuerte orientación a la adquisición del conocimiento tecnológico externo.

Resultará interesante profundizar la investigación sobre como las particularidades de cada entorno regional determinan el efecto que las capacidades tecnológicas generan en el sistema productivo. Ello permitirá entender cómo la estructura económica determina las relaciones que se establecen entre sectores y empresas y su impacto en el desempeño económico, la cr y el bienestar.

\section{REFERENCIAS BIBLIOGRÁFICAS}

Aboites, Jaime, y Dutrénit, Gabriela (2003), Innovación, aprendizaje y creación de capacidades tecnológicas, México, Miguel Ángel Porrúa/Uam Xochimilco.

Aboites, Jaime, y Soria, Manuel (2008), Economía del conocimiento y propiedad intelectual. Lecciones para la economía mexicana, México, Siglo xxi/Uam Xochimilco. Acs, Zoltan; Audretsch, David; Braunerhjelm, Pontus, y Carlsson, Bo (2004), “The missing link: the knowledge filter and entrepreneurship in economic growth", CEPR Working Paper 4783.

Aghion, Phillipe, y Howitts, Peter (1992), “A model of growth through creative destruction”, Econometrica, 60 (2), pp. 323-351.

Albornoz, Mario (2009), "Indicadores de innovación: las dificultades de un concepto en evolución”, Ciencia, Tecnología y Sociedad, 13 (5), pp. 9-25.

Archibugui, Daniele; Denni, Mario, y Filipetti, Andrea (2009), The global innovation 
scoreboard 2008: the dynamic of the innovative performance of countries, Bruselas, European Commission/DG Enterprises.

Archibugui, Daniele, y Coco, Alberto (2004), “A new indicator of technological capabilities for developed and developing countries (ArCo)", World Development, 32, (4), pp. 629-654.

Archibugui, Daniele, y Michie, Jonathan (1997), Technology globalisation and economic performance, UK, Cambridge University.

Audretsch, David; Keilbach, Max, y Lehmann, Erik (2006), Entrepeneurship and economic growth, New York, Oxford University Press.

Autio, Erkko; Kenney, Martin; Mustar, Phillipe; Siegel, Dan, y Wrigth, Mike (2014), "Entrepeneurial innovation: the importance of context", Research Policy, 0 (43), pp. 1097-1108.

Ayala, José (2005), Economía del sector público mexicano, México, Grupo Editorial Esfinge.

Blaug, Mark (1963), “A survey of the theory of process-innovations”, Economica, 30 (117), pp. 13-32.

Buesa, Mikel; Heijs, Joost; Martínez, Mónica, y Baumert, Thomas (2004), “Configuración estructural y capacidad de producción de conocimiento en los sistemas regionales de innovación: un estudio del caso español", IAIF, documento de trabajo 45 .

Chang, Ha-Joon (1993), "The political economy of industrial policy in Korea", Cambridge Journal of Economics, 0 (17), pp. 131-157.

Cimoli, Mario (2000), Developing Innovation Systems. Mexico in a Global Context, London, Continuum.

Cimoli, Mario; Porcile, Gabriel; Primi, Annalisa, y Vergara, Sebastián (2005), "Cambio estructural, heterogeneidad productiva y tecnología en América Latina”, en M. Cimoli (edit.), Heterogeneidad estructural, asimetrías tecnológicas y crecimiento en América Latina, Chile, CEPAL/BID.

Cimoli, Mario, y Dosi, Giovanni (1994), "De los paradigmas tecnológicos a los sistemas nacionales de producción e innovación”, Comercio Exterior, 44 (8), pp. 669-682.

Engle, Robert, y Granger, Clive (1987), "Co-integration and error correction: representation, estimation and testing", Econometrica, 0 (55), pp. 251-276.

Fagerberg, Jan; Srholec, Martin, y Verspagen, Bart (2010), "Innovation and economic development", en B.H. Hall y N. Rosenberg, Handbook of economics of innovation, vol. 2, Amsterdam, Elsevier.

Freeman, Christopher, y Pérez, Carlota (1988), Structural crises of adjustment, business cycle and investment theory, London, Pinter. 
Furman, Jeffrey; Porter, Michael, y Stern, Scott (2002), "The determinants of national innovative capacity", Research Policy, 0 (31), pp. 899-933.

Griliches, Zvi (1979), R\&D and Productivity: The Econometric Evidence, USA, University of Chicago Press.

Grossman, Gene, y Helpman, Elhanan (1991), Innovation and Growth in the Global Economy, Cambridge, The MIT Press.

Heston, Alan; Summers, Robert, y Aten, Betina (2012), Penn World Table Version 7.1, s.l., Center for International Comparisons of Production, Income and Prices, University of Pennsylvania.

Jaffe, Adam, y Trajtenberg, Manuel (2002), Patents, citations and innovations. A window on the knowledge economy, Massachusetts, The MIT Press.

Loasby, Brian J. (2001), "Time knowledge and evolutionary dynamics: why connections matter", Journal of Evolutionary Economics, 11 (4), pp. 393-412.

López-Leyva, Santos; Castillo-Arce, Miriam; Ledezma-Torres, José, y Ríos-Flores, Jesús (2014), "Economic growth from a theoretical perspective of knowledge economy: an empirical analysis for Mexico", Management Dynamics in the Knowledge Economy, 2 (2), pp. 217-239.

Lundvall, Beng-Ake (2010), National systems of innovation: toward a theory of innovation and interactive learning, London, Anthem Press.

Martínez, Mónica, y Baumert, Thomas (2003), "Medida de la capacidad innovadora de las comunidades autónomas españolas: construcción de un índice regional de la innovación", IAIF, documento de trabajo 35.

Metcalfe, John Stanley (2002), "Knowledge of growth and the growth of knowledge", Journal of Evolutionary Economics, 12 (1), pp. 3-15.

Mungaray, Alejandro (1994), "Paradigmas de organización industrial y posibilidades de innovación en las pequeñas empresas: análisis de enfoques y experiencias”, Investigación Económica, 54 (209), pp. 249-284.

Mungaray, Alejandro; Ramírez, Natanael, y Texis, Michell (2005), "Estructura de mercado y maximización de beneficios en las microempresas", Comercio Exterior, 55 (4), pp. 316-321.

Mungaray, Alejandro, y Palacio, Juan I. (2000), "Schumpeter, la innovación y la política industrial”, Comercio Exterior, 50 (12), pp. 1085-1089.

Nelson, Richard (1994), "The co-evolution of technology, industrial structure and supporting institutions", Journal industrial and corporate change, 3 (1), pp. 47-63.

Nelson, Richard, y Winter, Sidney (1982), An evolutionary theory of economic change, Cambridge, The Belknap Press of Harvard University Press.

Oster, Sharon (2000), Análisis moderno de la competitividad, México, Oxford. 
oCDE y Eurostat (2005), Manual de Oslo. Guía para la interpretación y recogida de datos sobre innovación [pdf]. Consultado el 16 de mayo de 2013, en: http://www. uis.unesco.org/Library/Documents/OECDOsloManual05_spa.pdf.

Phelps, Edmund (1962), "The new view of investment: a neoclassical analysis", Quaternary Journal of Economics, 76 (4), pp. 548-567.

Pérez, Carlota (1986), “Las nuevas tecnologías: una visión de conjunto”, Estudios Internacionales, 0 (76), pp. 420-459.

Pérez, César (2006), Análisis multivariante con aplicaciones para SPSS 15, España, Prentice Hall.

Porter, Michael, y Stern, Scott (2010), National Innovative Capacity [pdf]. Consultado el 18 de octubre de 2011, en: http://www.isc.hbs.edu/Innov_9211.pdf.

RICYT (2001), Normalización de indicadores de innovación tecnológica en América Latina y el Caribe. Manual de Bogotá [pdf]. Consultado el 15 de octubre de 2011, en: http://www.uis.unesco.org/Library/Documents/Bogota\%20Manual_Spa.pdf.

Ríos, Jesús, y Castillo, Miriam (2013), "La capacidad innovadora y el desempeño económico y tecnológico de México”, en J. C. Bermúdez (edit.), Emprendimiento e innovación para la competitividad internacional. Dimensiones, factores y esquemas empíricos sobre sus causas y efectos, Costa Rica, Escuela de Relaciones Internacionales de la Universidad Nacional.

Romer, Paul M. (1990), "Endogenous technological change”, Journal of Political Economy, 98 (5), pp. 71-102.

Rosenberg, Nathan (1999), Inside the black box: technology and economics, New York, Cambridge University Press.

Ruiz, Clemente (2008), “México: geografía económica de la innovación”, Comercio Exterior, 58 (11), pp. 756-768.

Sala-i-Martin, Xavier (2000), Apuntes de crecimiento económico, España, Antoni Bosch. Sánchez, Yesenia; García, Francisco, y Mendoza, Esteban (2014), "Determinantes de la capacidad innovadora regional en México. Una tipología de las regiones”, Región y Sociedad, 24 (61), pp. 119-160.

Schumpeter, Joseph (1912), The theory of economic development, Cambridge, Harvard University Press.

— (1928), “The instability of capitalism", The Economic Journal, 38 (151), pp. 361-386.

(1939), Business Cycles, New York, McGraw Hill.

Sener, Sefer, y Saridogan, Erran (2011), "The effects of science-technology-innovation on competitiveness and economic growth", Social and Behavioral Sciences. 0 (24), pp. 815-825. 
36 ECONOMÍA: TEORÍA Y PRÁCTICA • Nueva Época, número 43, julio-diciembre 2015

Soete, Luc; Verspagen, Bart, y Baster, Weel (2010), "Systems of innovation", en B. H. Hall y N. Rosenberg, Handbook of economics of innovation, vol. 2, Amsterdam, Elsevier.

Solow, Robert (1957), "Technical change and the aggregate production function", The Review of Economics and Statistics, 39 (3), pp. 312-320.

- (1969), "Investment and technical change", en K. Arrow, S. Karlin y P. Suppes (eds.), Mathematical Methods in the Social Sciences, Palo Alto, Stanford University Press.

Stern, Scott; Porter, Michel, y Furman, Jeffrey (2000), "The Determinants of National Innovative Capacity”, NBER Working Paper 7876 [en línea]. Consultado el 15 de septiembre de 2011, en: http://www.nber.org/papers/w7876.

Valdez-Lafarga, Cuitláhuac, y León-Balderrama, Jorge (2015), "Hacia una taxonomía de los sistemas regionales de innovación en México", Economía, Sociedad y Territorio, 15 (48), pp. 517-553. 